\title{
The effect of timing and spatial separation on the velocity of auditory apparent motion
}

\author{
THOMAS Z. STRYBEL, SHERRY A. SPAN, and APRIL M. WITTY \\ Califormia State University, Long Beach, Califormia
}

\begin{abstract}
Previously, it was shown that the minimum conditions for the illusion of auditory apparent motion (AAM) depend on stimulus timing but not spatial separation. In the present experiment, the effects of stimulus timing and source separation on the perceived velocity of AAM were examined. Eight listeners estimated the velocity, duration, and distance traveled of AAM, using a no-modulus, magnitude estimation procedure. Four burst durations $(25,50,100$, and $300 \mathrm{msec}), 10$ stimulus onset asynchronies (SOAs; 30, 40, 50,60, 70, 80, 90, 100, 110, and $120 \mathrm{msec}$ ) and two separations $\left(10^{\circ}\right.$ and $\left.40^{\circ}\right)$ were tested. Perceived velocity estimates were related to the total duration (burst duration $+\mathrm{SOA}$ ) of the stimulus sequence. The effect of separation on velocity was extremely small but statistically significant. These results are similar to those obtained previously on the minimum conditions for AAM. Duration estimates were related only to total duration, but separation estimates were related to both separation and total duration. These results suggest that velocity is possibly a primary dimension of AAM that is independent of source separation.
\end{abstract}

One of the most difficult problems facing the psychophysicist is stimulus definition (Stevens, 1951), and nowhere is this problem more difficult than in the field of motion perception. Although motion can be perceived in all sense modalities having the capability to detect the spatial properties of objects, a precise definition of the stimulus for motion perception continues to elude researchers (e.g., Kolers, 1972). Physical motion is defined as a change in an object's location over time. When one attempts to relate perceived motion to this physical definition, problems may arise, because motion can be perceived when an object's physical location does not change (see, e.g., Anstis, 1970).

The stimulus for motion perception is more difficult to define when perceived velocity is considered. Physical velocity is defined as the ratio of the extent of travel to the duration of the movement. Because physical velocity depends on two parameters, some have argued that perceived velocity is inferred from estimates of distance traveled and duration of movement. In this view, velocity perception requires two inputs: extent of travel and duration of movement. On the other hand, others have suggested that velocity is a primary sensation and not inferred from time and distance estimates. The stimulus, in this view, cannot be described in terms of distance and time. Investigators of visual velocity perception have been unable to resolve which of these two possibilities accurately describes the

The production of this publication was partially funded by a grant from the California State University Long Beach Foundation. We thank Associate Editor Bruce A. Schneider and three anonymous reviewers for their comments on previous versions of this manuscript. Correspondence concerning this article should be addressed to T. Z. Strybel, Department of Psychology, California State University, Long Beach, Long Beach, CA 90840 (e-mail: tstrybel@csulb.edu). perception of velocity. Proponents of velocity as a primary sensation (see, e.g., Lappin, Bell, Harm, \& Koitas, 1975; Orban, DeWolf, \& Maes, 1984) argue that velocity discrimination is more accurate than would be predicted from measures of distance and time discrimination. Furthermore, the existence of velocity-tuned cells in the cat and monkey (Maunsell \& Van Essen, 1983; Orban, Kennedy, $\&$ Maes, 1981) and velocity adaptation (see, e.g., Goldstein, 1957) are said to support the primary velocity view (Lappin et al., 1975). Opponents of velocity as a primary sensation argue that, if velocity is perceived directly, two stimuli having the same velocity yet differing in distance traveled and duration of movement should be perceived as having equivalent velocities, yet they are not (see, e.g., Brown, 1931).

Algom and Cohen-Raz $(1984,1987)$ provide yet another view of velocity perception. They argue that perceived velocity will be affected by distance traveled and travel time, whether it is a primary sensation or inferred from time and distance estimates. Moreover, if velocity is inferred, it does not have to be predictable from subjective estimates of time and distance, because the subjective velocity estimates may not be linearly related to the internal representation of distance and time. Algom and CohenRaz showed that velocity estimates of moving stimuli were significantly affected by an interaction between duration and distance in a manner consistent with a ratio model of distance-time integration. When subjects made velocity judgments from static duration and distance information (cognitive velocity), the magnitude estimates were consistent with a subtractive (distance minus time) model. Haussman (1995) showed that estimates of visual velocity can be predicted from subjective estimates of duration and distance traveled, if tau and kappa effects are taken into account. Tau and kappa effects are produced 
when subjects judge either the spatial or the temporal separation of two stimuli presented in succession. The kappa effect refers to an increase in the perceived temporal separation between two stimuli as the spatial separation of the sources increases. The tau effect refers to an increase in the perceived spatial separation as the temporal separation increases (Jones \& Huang, 1982). Haussman had observers estimate duration, distance traveled, and velocity in separate experiments. Velocity estimates predicted from the ratio of perceived distance traveled to perceived duration were more accurate than velocity estimates predicted from the power function relating perceived to physical velocity. In Haussman's model, the effects of distance traveled on perceived duration (kappa effect) and duration on perceived distance traveled (tau effect) were factored in.

As this brief summary indicates, some controversy remains in the visual literature over the nature of the velocity-processing mechanism and the precise definition of the stimulus for velocity perception. Moreover, the relationship between perceived distance traveled, duration, and velocity may be considerably more complex than the relationship that describes physical velocity.

\section{Auditory Velocity Perception}

The difficulties encountered in defining the stimulus for motion are not limited to visual stimuli, and the debate over the nature of velocity perception has been extended to other modalities. In the field of auditory motion perception, physical motion is also defined as the change in a sound's location over time. Nevertheless, motion in the auditory modality, as in the visual modality, can be heard under conditions that contradict the definition of simple physical motion (see, e.g., Perrott \& Strybel, 1997; Strybel, 1994). These conditions were produced with an illusion of motion known as auditory apparent motion (AAM), which is the auditory counterpart to visual apparent motion. In the visual modality, apparent motion is created by the proper timing and placement of two discrete stimuli. Stimulus timing is measured by the duration of each stimulus and the stimulus onset asynchrony (SOA), the time between the onsets of the lead and lag stimuli. Placement refers to the location and separation between the stimuli. Timing and separation should have an equal impact on the illusion, on the basis of the physical definition of motion. In vision, apparent motion is affected by all these variables, and the combinations of duration, SOA, and separation that produce visual apparent motion are described by Kortes' laws (Kolers, 1972; Korte, 1915).

In audition, apparent motion is affected by stimulus timing but not by spatial separation (for a review, see Perrott \& Strybel, 1997). AAM can be heard at the same burst duration-SOA combinations for separations between $3^{\circ}$ and $160^{\circ}$, under monaural and binaural listening conditions and for sources separated in the horizontal and midsaggital planes (Strybel, Manligas, Chan, \& Perrott, 1990; Strybel \& Neale, 1994; Strybel, Witty, \& Perrott, 1992). In fact, motion can be heard when the lead and lag stimulus are presented from a single loudspeaker (Strybel, 1994; Perrott \& Strybel, 1997). The detection of AAM is affected by burst duration and SOA, however. The minimum burst duration for the illusion is roughly $25 \mathrm{msec}$ (Strybel \& Neale, 1994; Strybel et al. 1992), and the SOAs that produce AAM increase with burst duration, according to Korte's second law of visual apparent motion (Briggs \& Perrott, 1972; Strybel \& Neale, 1994). Although only stimulus timing affects the minimum conditions for motion, both spatial and temporal factors affect discrimination of the direction of motion. Reliable performance on direction of motion discrimination requires that the separation exceed the minimum audible angle. Performance on this task is also affected by listening condition (monaural vs. binaural), plane of separation (horizontal vs. midsaggital), and stimulus timing (Strybel et al., 1990; Strybel, Manligas, \& Perrott, 1989; Strybel \& Neale, 1994; Strybel et al., 1992).

The ineffectiveness of spatial separation on AAM is surprising, given that separation does affect the visual illusion and that physical motion by definition requires a change in both time and space. One possible explanation for these findings is that separation affects only the velocity of AAM. If the travel time of AAM is equivalent to SOA and the extent of travel equivalent to separation, AAM produced at larger separations should be heard as traveling faster. For example, SOAs of 40-60 msec will produce the perception of a continuously moving sound when the burst duration is $50 \mathrm{msec}$ for separations of $6^{\circ}$ to $160^{\circ}$ (Strybel et al., 1990). If a sound image travels $160^{\circ}$ in the same time that it travels $6^{\circ}$, the former image should be heard as moving faster. The purpose of the present investigation was to examine this possibility. Magnitude estimates of the velocity of AAM were measured for combinations of burst duration, SOA, and spatial separation with the sound sources in the horizontal plane.

Very little is known about the ability of human listeners to process real or apparent auditory velocity, however. Only two components of velocity perception have been investigated, velocity discrimination and velocity estimation. Auditory velocity difference limens (DLs) have been measured when stimulus duration is held constant (Altman \& Viskov, 1977; Grantham, 1986). Velocity DLs depend only on discrimination of distance traveled, implying that velocity is not directly perceived in audition (Grantham, 1986; Middlebrooks \& Green, 1991). This argument is, of course, a restatement of the inferential view of visual velocity processing, summarized above. Magnitude estimates of the velocity of moving sound sources are directly proportional to source velocity, with a slope of approximately one (Waugh, Strybel, \& Perrott 1979). The slope of the velocity function is not affected by the duration of the stimulus, but accuracy of the velocity estimates is affected by duration. Listeners underestimate velocity at durations between 30 and $100 \mathrm{msec}$ and are quite accurate at longer durations (Perrott, Buck, Waugh, \& Strybel, 1979). Because the data on auditory velocity perception is extemely sparse, in the present experiment 
we also obtained estimates of the duration and separation between the sources to determine whether the velocity of AAM could be predicted from these estimates.

\section{METHOD}

\section{Subjects}

Eight volunteers ( 5 males and 3 females) participated in this study. All the subjects were screened for normal hearing and had thresholds within $20 \mathrm{~dB}$ of audiometric zero at frequencies between 500 and $8000 \mathrm{~Hz}$. One subject had participated in previous apparent motion experiments. None had any experience estimating the velocity of either really or apparently moving sounds.

\begin{abstract}
Apparatus
The study was conducted in a $2.7 \times 3.7 \times 2.1 \mathrm{~m}$ semianechoic, audiometric chamber. The subjects sat in a chair in the center of a room with their heads fixed by a chin rest. Three Radio Shack Minimus-7 loudspeakers were mounted $1.12 \mathrm{~m}$ in front of the subject at an elevation corresponding to ear level. The loudspeakers were placed at $-10^{\circ}, 0^{\circ}$, and $30^{\circ}$ azimuth and remained stationary throughout the experiment. A black acoustically transparent curtain was placed in front of the loudspeakers to conceal their location from the subject. The stimulus, consisting of high-pass noise with a lower cutoff of $2 \mathrm{kHz}$ and rise/decay time of less than $1 \mathrm{msec}$, was produced by three Quatech WSB-12A waveform synthesizers and WSB-100 signal generators mounted in the expansion slots of an IBM PC/AT compatible computer. The output of each synthesizer was led through separate channels of two custom-made amplifiers. The level of each stimulus was set at $60 \mathrm{~dB}$ A-weighted, measured at the position of the subject's head.
\end{abstract}

\section{Procedure}

Each subject completed three no-modulus magnitude estimation tasks: velocity, duration, and separation. Each estimation task had a separate set of instructions that the subjects read before beginning. The subjects were informed that there were no correct or incorrect answers and instructed to take as much time as they needed to complete the task. For the velocity estimation task, the subjects listened to the stimulus and wrote on an answer sheet their velocity estimate of the moving sound in miles per hour ( $\mathrm{mph}$ ), a measure chosen because subjects can reliably report auditory or visual velocity with this scale (Waugh et al., 1979). If, on any trial, motion was not perceived, they were instructed to enter $0 \mathrm{mph}$. For the duration estimation task, the subjects listened to the stimulus and wrote on an answer sheet their duration estimate of the sound sequence. For the separation estimation task, the subjects estimated the distance traveled by the sound sequence. If the sound sequence did not move, they were told to estimate the distance between the two stationary sounds. For both duration and separation estimation, the subjects were instructed to make numerical estimates that were proportional to the magnitude of each event, but no units were provided for the estimate.

Four burst durations $(25,50,100$, and $300 \mathrm{msec}), 10$ SOAs ( 30 , $40,50,60,70,80,90,100,110$, and $120 \mathrm{msec}$ ) and two spatial separations $\left(10^{\circ}\right.$ and $\left.40^{\circ}\right)$ were tested within a session. The location of a pair of speakers used to present the stimulus sequence determined the separation. A $10^{\circ}$ separation was produced by presenting the lead stimulus through the speaker at $-10^{\circ}$, and the lag stimulus through the speaker at $0^{\circ}$. A $40^{\circ}$ separation was produced by presenting the lead and lag stimuli through speakers at $-10^{\circ}$ and $30^{\circ}$, respectively. Thus, the starting location of the stimulus was constant throughout the experiment, and the direction of motion was always left-right.

Each of the 80 unique combinations of burst duration, SOA, and separation were presented in random order twice, creating a trial block of 160 trials. A trial block lasted $10-12 \mathrm{~min}$. In the velocity estimation task, each subject completed five trial blocks, producing a total of 10 estimates for each stimulus combination. Only three trial blocks were run on the duration and separation estimation tasks, producing a total of 6 estimates per stimulus combination. This was done to reduce the time required of each subject. Each subject performed the velocity estimation task first, followed by the duration and separation estimation tasks, with the order of the final two tasks counterbalanced. When the subjects performed the velocity estimation task, they were unaware that they would be subsequently estimating duration and separation. This was done to avoid biasing the strategies used to estimate velocity. The 11 sessions required of each subject were run over a period of several days.

\section{RESULTS}

Before the velocity estimates were analyzed, the percentage of motion reports (velocity estimates greater than zero) was calculated. A repeated measures analysis of variance (ANOVA) produced a significant interaction between burst duration and SOA $[F(27,189)=12.18, p<$ $.001]$, as is shown in Figure 1. Fewer motion reports were obtained at SOAs less than $70 \mathrm{msec}$ for the 300 -msec burst duration and at SOAs greater than $100 \mathrm{msec}$ for the shorter burst durations $(25-100 \mathrm{msec})$. The significant interaction is consistent with Korte's second law of apparent motion, which states that the SOAs that produce motion increase with burst duration, although at a slower rate (Kolers, 1972; Korte, 1915). This relationship has been demonstrated previously in the auditory modality (see, e.g., Briggs \& Perrott, 1972). Consistent with our previous research on AAM (Strybel et al., 1990; Strybel et al., 1989; Strybel \& Neale, 1994; Strybel et al, 1992), separation had no effect on AAM, and motion was heard most often when the two sound events either overlapped or followed closely in time.

Three subjects reported no motion at some extreme burst duration-SOA combinations, and 3 more had less than $50 \%$ motion reports at some of these burst durationSOA combinations. Therefore, all subsequent analyses were performed only on SOAs between 50 and $90 \mathrm{msec}$, where motion was heard on at least half the trials by all the subjects at all burst durations and separations. In addition, all 0 -mph responses were eliminated before the geometric means of the velocity estimates were computed for each subject. ${ }^{1}$ A repeated measures ANOVA was performed on the log-transform of the velocity estimates. This transform was used to correct for violations of the equal-variance assumptions usually found with magnitude estimates (Stevens, 1975). All main effects were significant [SOA: $F(4,28)=5.91, p<.05$; separation: $F(1,7)=$ $5.64, p<.05$; and burst duration: $F(3,21)=26.36, p<$ $.0001]$, and all interactions were nonsignificant [SOA $\times$ separation: $F(4,28)=0.70, p=.60 ;$ SOA $\times$ burst duration: $F(12,84)=1.20, p=.44$; separation $\times$ burst duration: $F(3,21)=1.07, p=.38 ;$ SOA $\times$ separation $\times$ burst duration: $F(12,84)=0.43, p=.94]$. The effect of each variable on the mean velocity estimate is shown in Figure 2. Open symbols are for a $10^{\circ}$ separation, and filled symbols for a $40^{\circ}$ separation. The main effects of SOA and 


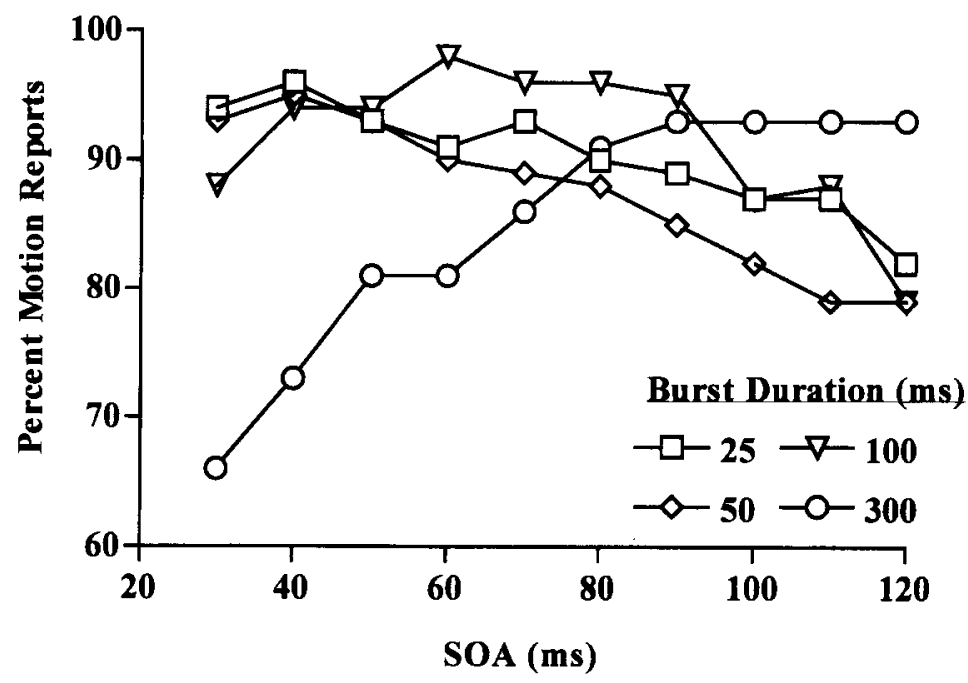

Figure 1. Percentage of motion reports (velocity estimates greater than zero) as a function of burst duration and stimulus onset asynchrony (SOA).

separation were small. SOA accounted for only $0.3 \%\left(\eta^{2}\right)$ of the total variance, and separation $0.1 \%$. As is shown in Figure $3 \mathrm{~A}$, the slope of the power function relating perceived velocity to SOA is only $-.13\left(r^{2}=.95\right)$. For separation, quadrupling the separation from $10^{\circ}$ to $40^{\circ}$ produced a small but consistent increase in velocity ( 37.7 to $40.4 \mathrm{mph}$ ). Burst duration, on the other hand, accounted for $20 \%$ of the variance, and, as is shown in Figure $3 \mathrm{~b}$, the slope of the power function was $-.23\left(r^{2}=.97\right)$. These results show that the perceived velocity of AAM is most influenced by burst duration, with SOA and separation having less effect. Given that the functions in Figure 2 are roughly parallel and the nonsignificance of all interactions, the effects of these variables on perceived velocity are strictly additive.

Figure 4 presents the effects of separation, burst duration, and SOA on the perceived duration of the stimulus sequence. An ANOVA was performed on the same levels of SOA as those used in the velocity analysis. Significant main effects of duration $[F(3,21)=70.13, p<.001]$ and

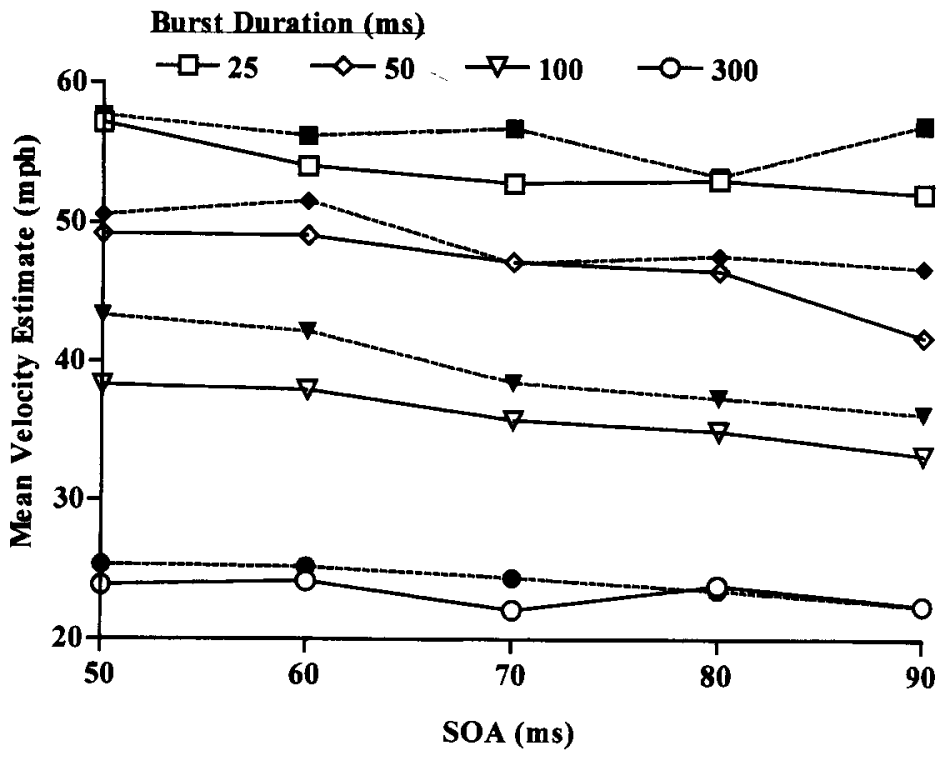

Figure 2. Geometric means of the velocity estimates of apparent auditory motion as a function of stimulus onset asynchrony (SOA), burst duration, and separation. Open symbols and solid lines represent $10^{\circ}$ separation. Filled symbols and dashed lines represent $40^{\circ}$ separation. 


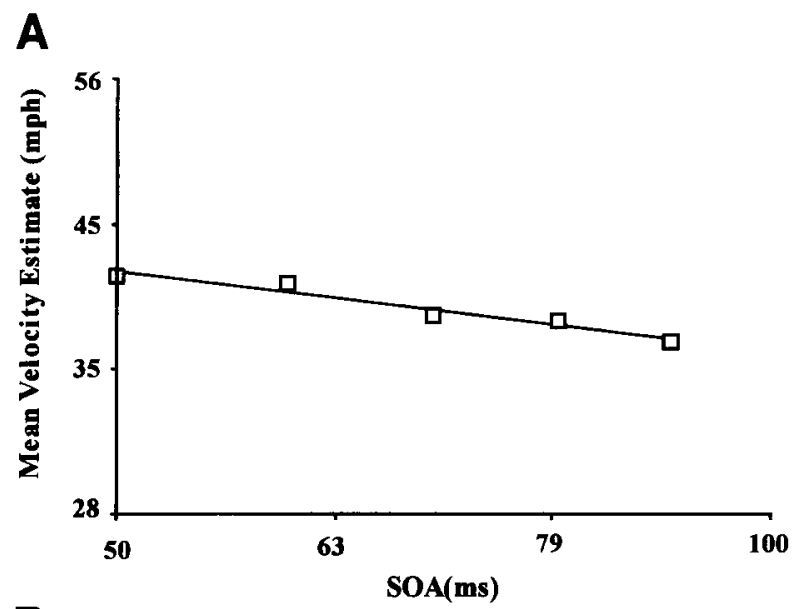

B

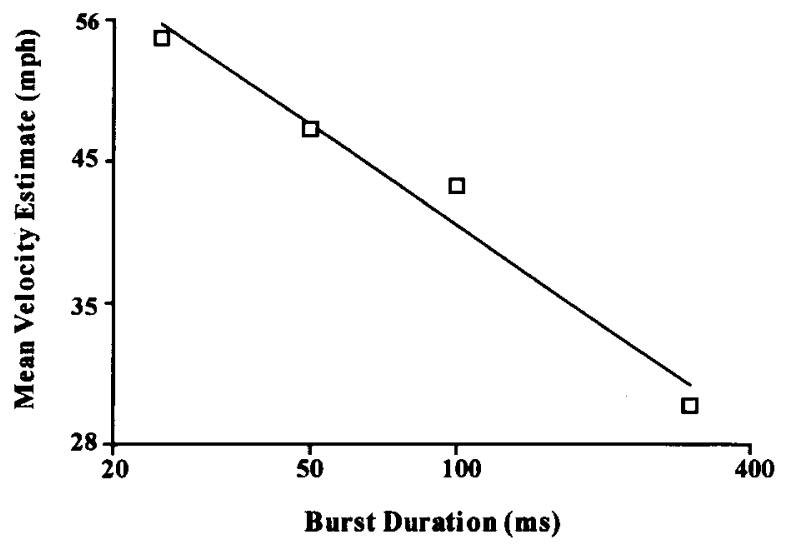

Figure 3. Geometric mean velocity estimates as a function of (A) stimulus onset asynchrony (SOA) and (B) burst duration, plotted in $\log -\log$ coordinates.

SOA $[F(4,28)=3.87, p<.02]$ were obtained, but neither separation nor any interaction was significant. As with the velocity estimates, burst duration accounted for more variance than did SOA $(28 \%$ vs. $0.23 \%)$. This difference in effectiveness is explained by the difference in relative contributions of burst duration and SOA to total duration. The difference in total duration produced by varying SOA at any one burst duration was only $40 \mathrm{msec}$, whereas the difference in total duration produced by varying burst duration at any one SOA was $275 \mathrm{msec}$. Burst duration simply contributes more to total duration than does SOA. The magnitude estimates of separation are presented in Figure 5. Not surprisingly, the main effect of separation was significant $\left[F(1,7)=9.27, p<.02, \eta^{2}=4.3 \%\right]$. A fourfold increase in the physical separation was heard as having traveled 1.5 times longer (average estimate 4.5 at $10^{\circ}$ vs. 6.3 at $40^{\circ}$ ). Somewhat surprising were the obtained main effects of burst duration $[F(3,21)=7.10, p<$ $\left..002 ; \eta^{2}=6.2 \%\right]$ and SOA $[F(4,28)=6.53, p<.0001$; $\left.\eta^{2}=0.23 \%\right]$. The strength of the effect of burst duration was slightly larger than the separation effect. Moreover, the slope of the power function relating burst duration to separation estimates was $.23\left(r^{2}=.99\right)$. Here, a fourfold increase in duration (e.g., from 25 to $100 \mathrm{msec}$ ) produced an increase of 1.4 (3.98 to 5.61). SOA was less effective in this regard (slope $=.17, r^{2}=.87$ ). No significant interactions were obtained on the separation estimates.

To summarize, the effects of burst duration, SOA, and separation on listeners' estimates of velocity were not equivalent. The perceived velocity of AAM is most affected by stimulus timing (burst duration and SOA). Although physical separation did significantly affect perceived velocity, the effect was extremely small. Estimates of duration were affected only by the variables (burst duration and SOA) that contribute to the total duration of the event. Given that the range of burst durations tested here was greater than the range of SOAs, duration estimates were affected more by burst duration than by SOA. Separation estimates, on the other hand, were affected equally by physical separation and stimulus timing, with burst duration again more effective than SOA.

Given the results shown in Figure 2, one would not expect velocity estimates to be highly related to the velocity model of AAM defined as the ratio of separation to SOA, because neither variable was as effective as burst duration. This is confirmed in Table 1, which presents the slopes and $r^{2}$ values for the power functions relating velocity, duration, and separation estimates to various models of velocity. When the mean velocity estimates were plotted against the ratio of separation to SOA (ratio model 1$)$, the fit was extremely poor $\left(r^{2}=.05\right)$, and the slope near zero. When velocity was modeled as the ratio of separation to total duration (ratio model 2), the fit was better $\left(r^{2}=.44\right)$ but still very poor. As is shown in Figure 6 , a separation of $40^{\circ}$ creates physical velocities that are four times those at $10^{\circ}$, yet the estimates of velocity increase much less. In fact, two separate functions are evident in this figure, one at each separation. The fit of the power functions relating velocity estimates to physical velocity at each separation are good $\left(r^{2}=.98\right.$ and .97 for $10^{\circ}$ and $40^{\circ}$, respectively), and the slopes are identical (.60).

Given the failure of ratio models of velocity, it is possible that the velocity estimates of AAM represented a linear sum of perceived duration and perceived distance traveled, as proposed by Algom and Cohen-Raz (1987) for cognitive velocity. It would be interesting to see whether the perceived apparent velocity judgments from the present experiment represent the perceived distance traveled minus the perceived time of travel. However, in order to make quantitative predictions, it is necessary to know the relative weight given to distance and travel time in the equation. Because these weights are unknown and cannot be determined in the present experiment, no quantitative evaluation of the cognitive model was attempted. However, it is likely that perceived distance traveled will receive a much lower weight than travel time, because of the very small contribution of physical separation to perceived velocity, as compared with the much larger contribution of total duration to perceived velocity. Indeed, 


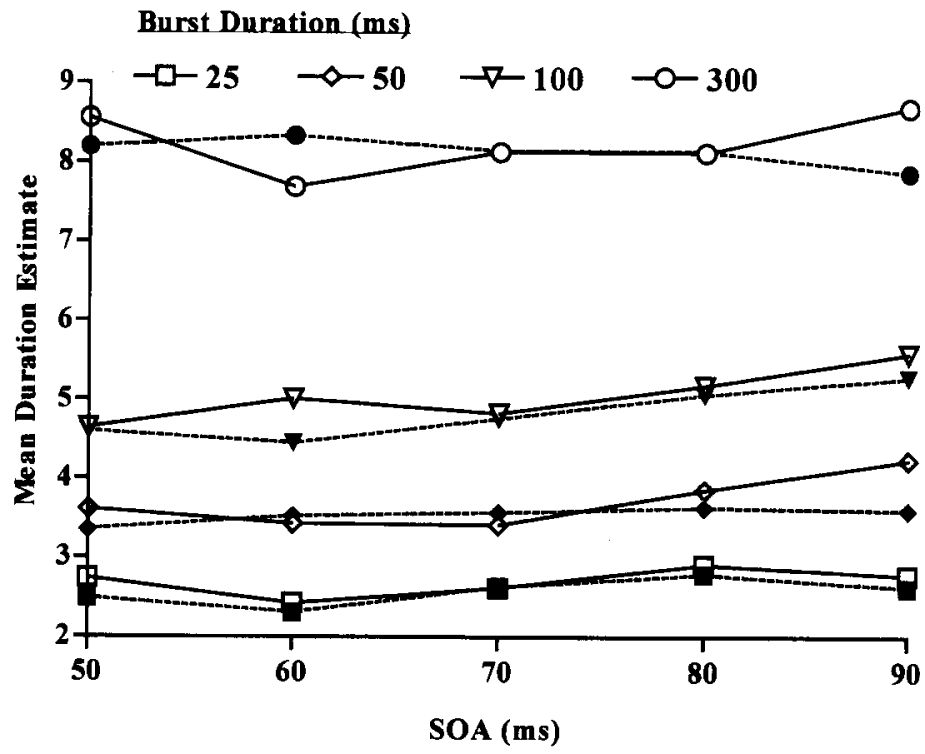

Figure 4. Geometric means of the duration estimates as a function of stimulus onset asynchrony (SOA), burst duration, and separation. Open symbols and solid lines represent $10^{\circ}$ separation. Filled symbols and dashed lines represent $40^{\circ}$ separation.

the best predictor of the obtained velocity estimates, shown in the bottom row of Table 1, is simply the total duration of the stimulus sequence $\left(r^{2}=.97\right)$. This relationship is shown in Figure 7 . The absolute value of the slope of the function $(-.6)$ is identical to the slopes obtained at each separation in Figure 6.

Slopes and $r^{2}$ values for the mean duration estimates are also presented in Table 1. Not surprisingly, the best fit was obtained with the total duration of the event $\left(r^{2}=\right.$ $.95)$. However, the slope of this function $(.78)$ is larger than the slope of the velocity function $(-.6)$. The difference between the absolute value of the velocity and duration slopes was marginally significant $[t(7)=1.79, p=$ .12 , two-tailed]. Determining the psychophysical function for separation estimates was less straightforward. As is shown in Table 1, the best predictor was also total

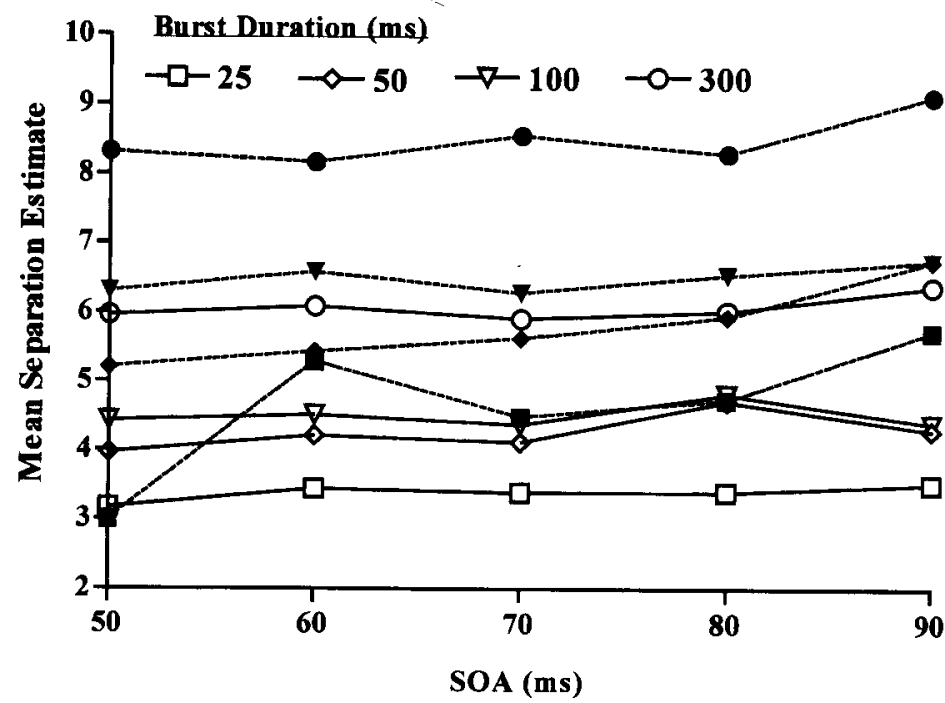

Figure 5. Geometric means of the separation estimates as a function of stimulus onset asynchrony (SOA), burst duration, and separation. Open symbols and solid lines represent $10^{\circ}$ separation. Filled symbols and dashed lines represent $40^{\circ}$ separation. 
Table 1

Power Function Exponents (Slopes) and Goodness-of-Fit $\left(r^{2}\right)$ Values for Various Models of Velocity, Duration, and Separation Estimation

\begin{tabular}{|c|c|c|c|}
\hline Estimation & $\begin{array}{c}\text { Ratio 1 } \\
\text { (Separation/Stimulus Onset Asynchrony) }\end{array}$ & $\begin{array}{c}\text { Ratio } 2 \\
\text { (Separation/Total Duration) }\end{array}$ & $\begin{array}{c}\text { Total Duration } \\
\text { (Stimulus Onset Asynchrony \& Burst Duration) }\end{array}$ \\
\hline \multicolumn{4}{|l|}{ Velocity } \\
\hline Slope & .01 & .24 & -.60 \\
\hline$r^{2}$ & .05 & .44 & .97 \\
\hline \multicolumn{4}{|l|}{ Duration } \\
\hline Slope & -.04 & -.30 & .78 \\
\hline$r^{2}$ & .004 & .14 & .95 \\
\hline \multicolumn{4}{|l|}{ Separation } \\
\hline Slope & .20 & -.003 & .41 \\
\hline$r^{2}$ & .11 & .0001 & .58 \\
\hline
\end{tabular}

Note-Ratio models are in miles per hour. Total duration model is in milliseconds.

duration, but the fit was poor $\left(r^{2}=.58\right)$. In fact, no model adequately predicted estimates of separation. The difficulty can be explained by an examination of individual subject data presented in Table 2 and Figure 8 . In the third column of Table 2 , individual separation estimates are regressed against total duration. The estimates of three subjects (S3, S5, and S6) were highly related to total duration. S6's estimates of velocity, duration, and separation are presented in Figure 8A. In contrast, S2 and S7's separation estimates were based on separation only and were independent of total duration, as is shown in Figure $8 \mathrm{~B}$ for S7. Finally, three subjects (S1, S4, and S8) used both physical separation and total duration, as exemplified by S1's scatterplot in Figure 8C. Note, however, that the velocity and duration estimates for each subject shown in Figure 8 are affected only by total duration. We did not observe any individual differences in the effectiveness of timing and separation on either velocity or duration estimates. This is also shown by the consistently high $r^{2}$ values in columns 2 and 4 of Table 2 .

\section{DISCUSSION}

These results suggest that velocity is a separate and possibly primary dimension of AAM. The illusion is heard to move at different velocities, and human listeners can reliably scale its velocity. The functions relating velocity estimates to total duration were different from those obtained for either duration or distance traveled. Furthermore, stimulus timing was highly predictive of the per-

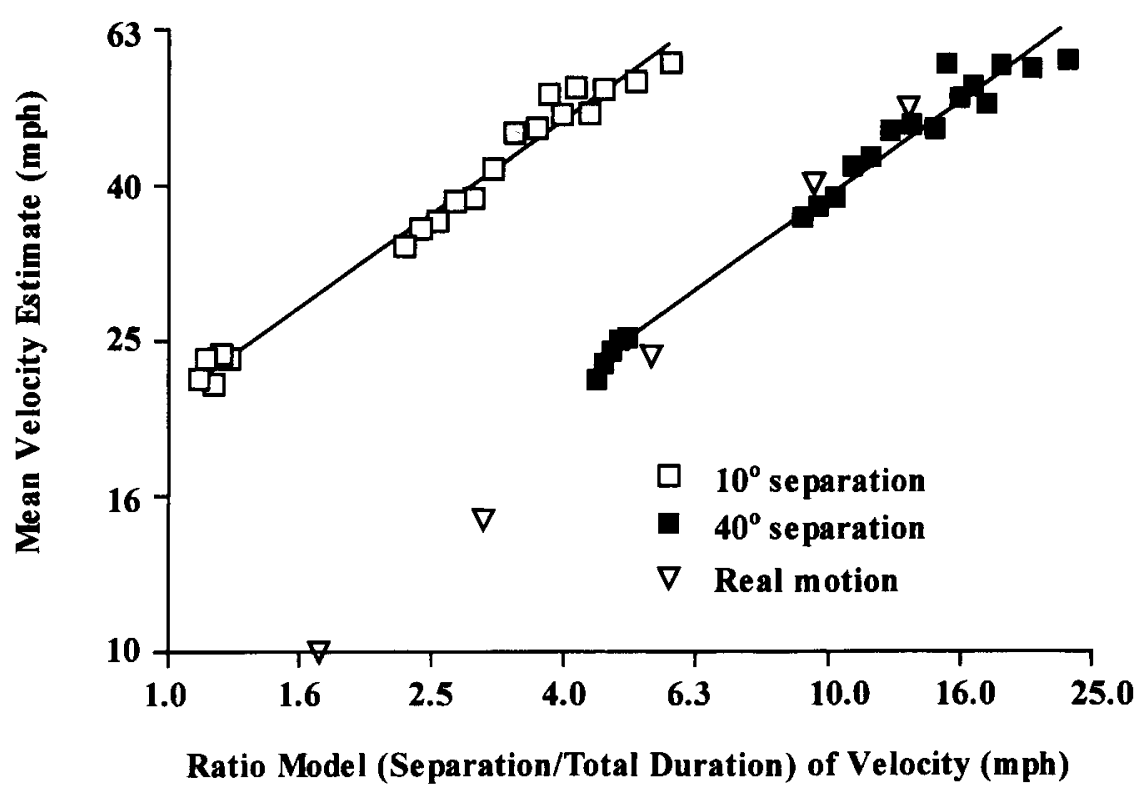

Figure 6. Geometric means of the velocity estimates of apparent auditory motion as a function of a ratio model (separation/total duration) of velocity in mph, plotted in log-log coordinates. Open squares represent $10^{\circ}$ separation, and filled squares represent $40^{\circ}$ separation. Real motion data is taken from Waugh et al. (1979). 


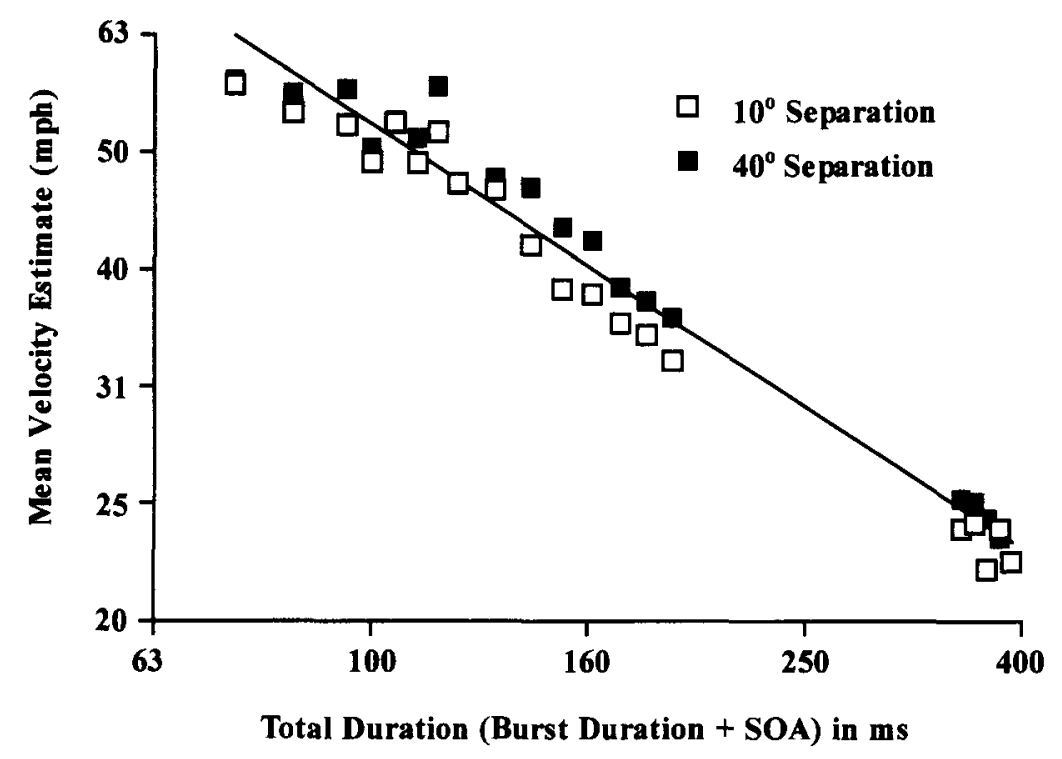

Figure 7. Geometric mean velocity estimates of apparent auditory motion as a function of the total duration (stimulus onset asynchrony [SOA] + burst duration) of the stimulus sequence, plotted in $\log$-log coordinates.

ceived velocity of AAM, more so than ratio models (separation/SOA or separation/total duration) of velocity perception. Our subjects were not inferring velocity from estimates of time and distance traveled. Although physical separation did affect velocity judgments, the effect was small. Some of the differences in sensitivity to timing and separation may be attributed to the fact that fewer values of separation were tested here, compared with the number of burst duration-SOA combinations. It would be interesting to determine whether the relative independence of velocity and separation found here would hold if a greater number of separations were used. However, distance traveled should have been easy to estimate, because the starting location and direction of travel were constant throughout the experiment. Even under these conditions, most listeners gave little weight to distance traveled when estimating velocity. These results imply that the perceived velocity of AAM is mediated by the same mechanism that determines the minimum conditions for the illusion, because both are determined almost ex- clusively by stimulus timing and not separation (see, e.g., Strybel et al., 1990; Strybel \& Neale, 1994; Strybel et al., 1992).

Previous research on auditory velocity perception either used an objective task (velocity discrimination) with simulated motion (see, e.g., Altman \& Viskov, 1977; Grantham, 1986) or obtained velocity judgments of physically moving sound sources (Perrott et al., 1979; Waugh et al., 1979). Altman and Viskov (1977) measured DLs for velocities between $14 \% \mathrm{sec}$ and $140 \%$ sec when dichotic motion was produced by dynamic variations in interaural time differences (ITDs). Velocity DLs were linearly related to the rate of change in ITD. Grantham (1986) measured DLs for reference velocities between $0 \% / \mathrm{sec}$ and $150 \% \mathrm{sec}$. In Grantham's experiment, the duration was constant throughout a trial block, meaning that faster moving sounds always traveled farther. The subjects could, therefore, discriminate on the basis of either velocity or extent of travel. Grantham showed that velocity DLs were based on detecting differences in extent of travel only

Table 2

Individual Power Function Exponents (Slopes) and Goodness-of-Fit $\left(r^{2}\right)$ Values for the Total Duration (Stimulus Onset Asynchrony + Burst Duration) Model

\begin{tabular}{|c|c|c|c|c|c|c|}
\hline \multirow[b]{2}{*}{ Participant Number } & \multicolumn{2}{|c|}{ Velocity Estimation } & \multicolumn{2}{|c|}{ Duration Estimation } & \multicolumn{2}{|c|}{ Separation Estimation } \\
\hline & Slope & $r^{2}$ & Slope & $r^{2}$ & Slope & $r^{2}$ \\
\hline 1 & -.44 & .92 & .56 & .79 & .24 & .1 \\
\hline 2 & -.44 & .79 & .62 & .96 & .58 & .0 \\
\hline 3 & -.57 & .76 & .64 & .87 & .66 & .75 \\
\hline 4 & -1.26 & .89 & 1.05 & .81 & .62 & 14 \\
\hline 5 & -.38 & .85 & .79 & .93 & .84 & .93 \\
\hline 6 & -.35 & .89 & .95 & .76 & .98 & 8 \\
\hline 7 & -.75 & .87 & .60 & .76 & -.05 & .01 \\
\hline 8 & -.66 & .92 & 1.01 & .88 & .28 & 30 \\
\hline
\end{tabular}



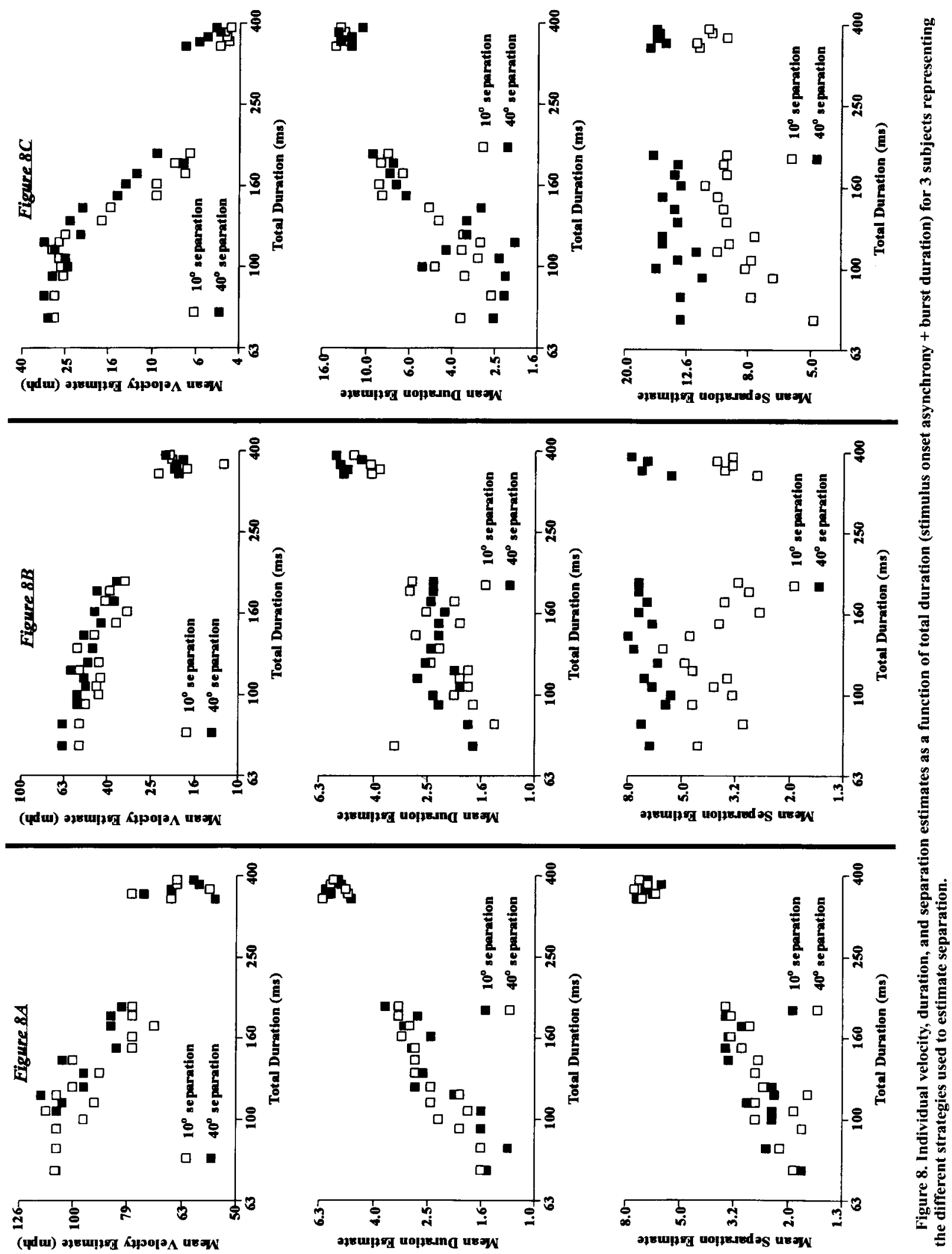
and asserted that velocity is not sensed directly in audition. Our results suggest, however, that, if duration was varied within a session, discrimination performance based on extent of travel would be poorer. In our experiment, a slow-moving or long-duration stimulus was heard to travel farther than a fast-moving or short-duration stimulus traversing the same physical distance. Furthermore, Grantham noted considerable intersubject variability in his experiment. This variability might be explained by our finding that subjects use different strategies to judge distance traveled.

Besides velocity discrimination, magnitude estimates of physically moving sound sources have been obtained. Magnitude estimates of velocity of real motion were directly related to physical velocity, with a slope of roughly 1 (Perrott et al., 1979; Waugh et al., 1979). In the present experiment, a power function was obtained, and the slope was nearly half (.60) that obtained for real motion. This difference is probably due to the limited range of velocities tested in our study. Whereas Waugh et al. used velocities between $15 \% \mathrm{sec}$ and $360 \% \mathrm{sec}$, illusory movement cannot be produced at low velocities, because AAM is not heard at long durations and SOAs. The values from Waugh et al. at velocities similar to those in the present study are reproduced in Figure 6 . The auditory real and the illusory function at $40^{\circ}$ separation overlap at higher velocities. This is, we believe, one of the first demonstrations of the equivalence of auditory real and apparent motion. It is also interesting that Perrott et al. found no effect of stimulus duration on the slopes of psychophysical functions. Given that longer stimulus durations produce greater extents of travel, extent of travel did not appear to affect perceived velocity in the Perrott et al. experiment, consistent with the results obtained in the present experiment on illusory motion.

The effect of stimulus timing on separation estimates was surprising. One might suggest that this finding is explained by the tau effect (Jones \& Huang, 1982), whereby estimates of the distance between two stationary stimuli presented in succession increase with the temporal separation between them. We would argue against this explanation, however, because tau and kappa effects are usually obtained with longer temporal separations (600$6,000 \mathrm{msec}$ ) and only for stationary stimuli. In fact, when the extent of visual apparent motion is estimated, a reverse of the tau effect can be obtained (Mashour, 1964). Here, separation estimates are inversely related to duration. Furthermore, in the present experiment, we did not see any evidence of the kappa effect. That is, estimates of the perceived duration of AAM were not affected by the spatial separation between the sources.

An alternative explanation of the effect of duration on perceived separation might be found in Perrott and $\mathrm{Mu}-$ sicant (1977). Listeners in one experiment indicated the perceived location of the start and endpoints of a physically moving sound source. Extent of travel estimates were sub- sequently computed from these estimates. Both duration $(50-300 \mathrm{msec})$ and velocity $\left(90 \% \mathrm{sec}-360^{\circ} / \mathrm{sec}\right)$ were manipulated. The apparent onset of the stimulus was displaced in the direction of motion, with the amount of displacement dependent on source velocity. The apparent offset of the stimulus depended on both duration and velocity. The duration of the pulse affected perceived extent of travel, with the path length of shorter duration pulses being overestimated and the path length of longer duration pulses underestimated. This fact that stimulus duration affected perceived extent of travel is consistent with our findings on estimates of separation. If separation or distance traveled is used in estimating velocity, it may not provide reliable information, because perceived extent of travel depends on both stimulus duration and velocity. Furthermore, estimates of separation in our experiment were highly dependent on the strategies used by different subjects. In fact, one might conclude on this basis that velocity is primary in audition and the extent of travel is inferred from perceived duration and velocity.

In summary, the velocity of AAM can be perceived directly and is affected primarily by the timing variables of burst duration and SOA but is independent of source separation. This finding is consistent with previous research examining the minimum stimulus conditions for AAM (see, e.g., Strybel et al., 1990; Strybel \& Neale, 1994; Strybel et al., 1992). The similarity of the velocity estimates obtained here to velocity estimates of really moving sound sources (Perrott et al., 1979; Waugh et al., 1979), as well as the similarity of the separation estimates of AAM to extent-of-travel estimates of really moving sounds (Perrott \& Musicant, 1977), suggests similarities between the processing of AAM and real motion. Moreover, AAM may tap into a high-velocity processing system, because the similarities between AAM and real motion were found with real motion at high velocities. The ineffectiveness of the distance traveled or separation would be reasonable at these velocities, given that the binaural system is insensitive to rapid changes in interaural time differences (see, e.g., Blauert, 1983; Grantham \& Wightman, 1978; Saberi \& Hafter, 1996). It is possible that AAM, which is only minimally affected by binaural cues (see, e.g., Strybel et al., 1989; Strybel \& Neale, 1994) may stimulate a mechanism that is specialized for processing highvelocity moving sound sources.

\section{REFERENCES}

Algom, D., \& Cohen-Raz, L. (1984). Visual velocity input-output functions: The integration of distance and duration onto subjective velocity. Journal of Experimental Psychology: Human Perception \& Performance, 10, 486-501.

Algom, D., \& Cohen-Raz, L. (1987). Sensory and cognitive factors in the processing of visual velocity. Journal of Experimental Psychology: Human Perception \& Performance, 13, 3-13.

Altman, J. A., \& Viskov, O. V. (1977). Discrimination of perceived movement velocity for fused auditory image in dichotic simulation. Journal of the Acoustical Society of America, 61, 816-819. 
ANstis, S. M. (1970). Phi movement as a subtraction process. Vision Research, 10, 1411-1430.

Blauert, J. (1983). Spatial hearing. Cambridge, MA: MIT Press.

Briggs, R., \& Perrott, D. R. (1972). Auditory apparent movement under dichotic listening conditions. Journal of Experimental Psychology, 92, 83-91.

Brown, J. F. (1931). The visual perception of velocity. Psychologische Forschung, 14, 249-268.

GolDSTEIN, A. G. (1957). Judgments of visual velocity as a function of length and observation time. Journal of Experimental Psychology, 54, 457-461.

Grantham, D. W. (1986). Detection and discrimination of simulated motion of auditory targets in the horizontal plane. Journal of the Acoustical Society of America, 79, 1939-1949.

Grantham, D. W., \& Wightman, F. L. (1978). Detectability of varying interaural temporal differences. Journal of the Acoustical Society of America, 63, 511 -523.

HaUSSMAN, R. E. (1995). The perceptual construction of visual velocity: Mechanism and model. Unpublished doctoral dissertation, Yale University.

JonEs, B., \& HUANG, Y. L. (1982). Space-time dependencies in psychophysical judgment of extent and duration: Algebraic models of the tau and kappa effects. Psychological Bulletin, 91, 128-142.

Kolers, P. A. (1972). Aspects of motion perception. Oxford: Pergamon.

KoRTE, A. (1915). Kinematoskopische Untersuchungen. Zeitschrift für Psychologie, 72, 193-206.

Lappin, J. S., Bell, H. H., Harm, O. H., \& KoItas, B. (1975). On the relations between time and space in the visual discrimination of velocity. Journal of Experimental Psychology: Human Perception \& Performance, 1, 383-394.

MASHOUR, M. (1964). Psychophysical relations in the perception of velocity. Stockholm: Almquist \& Wiksell.

Maunsell, J. H. R., \& Van Essen, D. C. (1983). Functional properties of neurons in the middle temporal visual area of the macaque monkey: I. Selectivity for stimulus direction, speed and orientation. Journal of Neurophysiology, 49, 1127-1147.

Middlebrooks, J. C., \& GREen, D. M. (1991). Sound localization by human listeners. Annual Review of Psychology, 42, 135-159.

Orban, G. A., DeWolf, J., \& MAEs, H. (1984). Factors influencing velocity coding in the human visual system. Vision Research, 24, 33-39.

Orban, G. A., Kennedy, H., \& Maes, H. (1981). Response to movement of neurons in areas 17 and 18 of the cat: Velocity sensitivity. Journal of Neurophysiology, 45, 1043-1058.

Perrott, D. R., Buck, V., Waugh, W., \& Strybel, T. Z. (1979). Dynamic auditory localization: Systematic replication of the auditory velocity function. Journal of Auditory Research, 19, 105-112.

Perrott, D. R., \& Musicant, A. D. (1977). Minimum audible movement angle: Binaural localization of moving sound sources. Journal of the Acoustical Society of America, 62, 1463-66.

PerrotT, D. R., \& Strybel, T. Z. (1997). Some observations regard- ing motion without direction. In R. Gilkey \& T. Anderson (Eds.), Binaural and spatial hearing in real and virtual environments (pp. 275294). Mahwah, NJ: Erlbaum.

SABERI, K., \& HAFTER, E. R. (1996). Experiments on auditory motion perception. In R. Gilkey \& T. Anderson (Eds.), Binaural and spatial hearing in real and virtual environments (pp. 315-327). Mahwah, $\mathrm{NJ}$ : Erlbaum.

STEvens, S. S. (1951). Mathematics, measurement and psychophysics. In S. S. Stevens (Ed.), Handbook of experimental psychology (pp. 1-49). New York: Wiley.

Stevens, S. S. (1975). Psychophysics. New York: Wiley.

STRYBEL, T. Z. (1994). The role of amplitude modulation in auditory motion perception [Abstract]. Journal of the Acoustical Society of America, 95(5, Pt. 2), 3004.

Strybel, T. Z., Manligas, C. L., Chan, O., \& Perrott, D. R. (1990). A comparison of the effects of spatial separation on apparent motion in the auditory and visual modalities. Perception \& Psychophysics, 47, 439-448.

Strybel, T. Z., Manligas, C. L., \& Perrott, D. R. (1989). Auditory apparent motion under binaural and monaural listening conditions. Perception \& Psychophysics, 45, 371-377.

Strybel, T. Z., \& Neale, W. N. (1994). The effect of burst duration, interstimulus onset interval and loudspeaker arrangement on auditory apparent motion in the free field. Journal of the Acoustical Society of America, 96, 3463-3475.

Strybel, T. Z., WitTy, A. M., \& PerrotT, D. R. (1992). Auditory apparent motion in the free field: The effects of stimulus duration and separation. Perception \& Psychophysics, 52, 139-143.

Waugh, W., Strybel, T. Z., \& Perrott, D. R. (1979). Perception of moving sounds: Velocity discrimination. Journal of Auditory Research, 19, 103-110.

\section{NOTES}

1. We instructed the subjects to report $0 \mathrm{mph}$ when no motion was heard, to prevent them from calculating the velocity of the event from static duration and separation information. In effect, we wanted to avoid cognitive velocity judgements (see, e.g., Algom \& Cohen-Raz 1984, 1987). When an ANOVA was performed on the full range of SOAs (including $0 \mathrm{mph}$ estimates), the only difference in the results was a significant interaction of burst duration and SOA. This interaction was due to lowered velocity estimates at extreme SOAs, caused by the $0 \mathrm{mph}$ judgments. No interactions with separation were obtained. Finally, the slopes of the velocity power functions were only minimally affected by the SOA range restriction. However, this restriction in SOA range increased the goodness-of-fit value.

(Manuscript received July 11, 1996; revision accepted for publication November 21, 1997.) 